\title{
Central Hepatectomy
}

National Cancer Institute

\section{Source}

National Cancer Institute. Central Hepatectomy. NCI Thesaurus. Code C91830.

A therapeutic surgical procedure to remove liver cancers located at the central segment of the liver. It involves the resection of the central hepatic segment with the left and right segments remaining intact. 